\title{
Generalized Uniform Singlet- and Triplet-Pair Extrapolation of the Correlation Energy to the One Electron Basis Set Limit
}

\author{
A. J. C. Varandas ${ }^{\dagger}$ \\ Departamento de Química, Universidade de Coimbra 3004-535 Coimbra, Portugal
}

Received: October 30, 2007; In Final Form: December 5, 2007

\begin{abstract}
The relationship between the triplet- and singlet-pair interaction coefficients in the uniform singlet- and tripletpair extrapolation method recently suggested for extrapolating $a b$ initio energies to the one-electron basis set limit is analyzed. Based on the premise that such a ratio is invariant over the configuration space of the molecule, generalizations of the method are investigated and their performance tested on extrapolations with MP2, CCD, CCSD, and MRCI(Q) energies. The best variant requires raw energies calculated using augmented correlated consistent basis sets with cardinal numbers up to $X=6$ at a single geometry. A scheme is also suggested that performs better than the traditional $X^{-3}$ law and possibly the original uniform singlet- and triplet-pair extrapolation method but requires data only up to $X=Q$.
\end{abstract}

\section{Introduction}

It is well-established that the conventional correlated orbitalbased methods involve only even powers of $r_{i j}$ while the exact wave function of an atomic or molecular system shows a linear dependence in the interelectronic coordinate $r_{i j}$ as this approaches zero. ${ }^{1,2}$ This is so whatever approach one uses to treat correlation effects [Møller-Plesset perturbation theory (MP), configuration interaction $(\mathrm{CI})$, coupled cluster (CC) method, etc], and explains the slow convergence of such methods with increasing number of Slater determinants used to represent the multi-electron wave function. In fact, this may explain why the highest levels of accuracy in electronic structure calculations have not been achieved via such methods but through unconventional ones such as the quantum Monte Carlo method ${ }^{3}$ or wave functions that depend explicitly on $r_{i j}{ }^{4-6}$ Yet, despite the rising success of the latter approaches, ${ }^{7}$ the conventional CI ansatz, the CC family of methods [CCD, CCSD, CCSD(T), CR$\mathrm{CC}(2,3)$, etc; for reviews, see ref 8 and a recent paper ${ }^{9}$ that gives references to such methods as applied to the potential energy surface (PES) of the water molecule] or MP2 perturbation theory ${ }^{8}$ continue to be routine especially when combined with extrapolation techniques. Indeed, the inclusion of $r_{i j}$ coordinates in multireference (MR) approaches has only recently been done using a novel variant of the explicitly correlated multireference averaged coupled-pair functional method and applied to the hydrogen fluoride molecule, ${ }^{10}$ with conventional CI methods [usually with the popular degenerate Davidson correction, $\mathrm{CI}(\mathrm{Q})$ or $\mathrm{MRCI}(\mathrm{Q})]$ proving essential for the calculation of accurate PESs. The same holds for CC methods which are known to treat electron correlation most efficiently, and we will examine here $\mathrm{CC}$ results obtained both with the inclusion of double excitations (CCD) and single and double excitations as well as perturbative corrections for connected triple excitations [CCSD and $\operatorname{CCSD}(\mathrm{T})$ ].

An enormous progress in electronic structure calculations for systems with small and moderate sizes arose with the introduction of correlated-consistent polarized valence basis sets ${ }^{11-14}$ (cc-pVXZ or $\mathrm{V} X \mathrm{Z}$ ), augmented ones (AVXZ) or even more

\footnotetext{
† E-mail address: varandas@qtvs1.qui.uc.pt.
}

flexible basis sets now being routinely employed. Built in a systematic manner that is intended to relate the correlation energy to the cardinal number $X$, such basis sets prompted the search for laws to extrapolate the total energy or its components to the complete basis set $(\mathrm{CBS})$ limit $^{8,15-28}$ (the list is by no means exhaustive), a subject also addressed in the present work.

Extrapolation to the CBS limit finds support on the dependence of the correlation energy on the partial wave quantum number for two-electron atomic systems and second-order pair energies in many-electron atoms. ${ }^{29-41}$ Specifically, the energy has been shown to vary as an inverse power of the cardinal number,

$$
E_{X}^{\mathrm{cor}}=E_{\infty}^{\mathrm{cor}}+\frac{A_{\mathrm{cor}}}{X^{\beta}}
$$

where $E_{X}^{\text {cor }}$ is the correlation energy for the basis set of cardinal number $X$, and $E_{\infty}^{\mathrm{cor}}, A_{\text {cor }}$, and $\beta$ are parameters. ${ }^{16,17}$ Asymptotically one expects the value of $\beta=3$, although Truhlar ${ }^{17}$ recommended optimal extrapolation exponents for MP2 $2^{42}\left(\beta_{\mathrm{MP} 2}\right.$ $=2.2), \operatorname{CCSD}$ and $\operatorname{CCSD}(\mathrm{T})\left[\beta_{\mathrm{CCSD}}=\beta_{\mathrm{CCSD}(\mathrm{T})}=2.4\right]$ calculations by minimizing the root-mean-square deviation (rmsd) in fits to Halkier et al. ${ }^{16}$ estimated basis-set limits for $\mathrm{Ne}, \mathrm{HF}$, and $\mathrm{H}_{2} \mathrm{O}$. Since his main interests were to develop a rule with potential interest for moderately large systems, such extrapolations focused on small basis sets ( $D$ an $T)$ and valenceonly limits, as for those systems changes in core correlation energy upon bond formation are usually small compared to other errors. ${ }^{43,44}$ The above numerical values have later been reevaluated by considering a larger set of reference data. ${ }^{18}$ Variable-exponent inverse-power laws have most recently been also utilzed by Bakowies, ${ }^{28}$ who used the adjustable $\beta$ parameter to quantify deviations from the asymptotic convergence behavior, and analyzed both analytical examples (i.e., other asymptotic expansions ${ }^{15,20,22,25,26}$ ) and numerical extrapolations to either large but finite or CBS targets. He observed that deviations from asymptotic convergence are most significant for extrapolations from small basis sets but still noticeable for basis sets as large as V5Z or V6Z. By further defining extrapolation exponents $\beta_{\text {opt }}(X, X+1, X+N)$ that are optimal for a given set of 
molecules, Bakowies ${ }^{28}$ suggested to vary $\beta$ such that the rmsd between extrapolated and explicitly calculated energies $E_{X+N}$ become minimal. Thus, rather than providing a single optimal exponent, he recommends specific exponents for specific extrapolations. His strategy is therefore similar to the one utilized by Schwenke, ${ }^{26}$ with the only formal difference being that the involved scaling is performed analytically rather than numerically. However, the most popular dual-level CBS law that warrants the correct asymptotic behavior is ${ }^{15}$

$$
E_{X}^{\mathrm{cor}}=E_{\infty}^{\mathrm{cor}}+\frac{A_{3}}{(X+\alpha)^{3}}
$$

where $E_{X}^{\text {cor }}$ and $A_{3}$ are parameters commonly determined from energies for the two highest affordable values of $X$, and the offset parameter $\alpha$ is fixed from some other condition. ${ }^{27}$ In fact, the potential benefit of using such rules is well recognized, ${ }^{45}$ justifying that the search for improved extrapolation techniques continues to be an active area of research. The reader is addressed to ref 45 for a detailed analysis of the sources of error in electronic structure calculations and a comparison of the performance of eq 2 with a few other rules on small chemical systems using $\mathrm{VXZ}$ basis sets.

Although also utilized for the total energy on the basis of the dominance of the correlation energy lowering (ref 46, and references therein), eq 2 finds its justification in the energy increments of partial-wave expansions of atomic correlation energie ${ }^{29-41}$ or similar expressions derived from the convergence behavior of the principal expansion. ${ }^{8,32,47}$ From a MP2 study on arbitrary excited states of He-like atoms, where the first-order wave function $\psi$ behaves for small $r_{12}$ as $\psi=(1+$ $\left.\kappa r_{12}\right) \Phi+Q\left(r_{12}^{2}\right)$ with $\kappa=1 / 2(1 / 4)$ for singlet (triplet) states ${ }^{1,2}$ and $\Phi$ being the HF wave function, Kutzelnigg and Morgan ${ }^{39}$ established the following: for natural-parity singlet states, the leading contribution at second-order of perturbation theory is proportional to $(\zeta+1 / 2)^{-4}$, with no contributing odd-terms proportional either to $(\zeta+1 / 2)^{-5}$ or $(\zeta+1 / 2)^{-7}$ and the term $(/$ $+1 / 2)^{-6}$ being universally $-(5 / 4)$ that of $(/+1 / 2)^{-4}$; for all triplet states, the leading term is proportional to $(/+1 / 2)^{-6}$; for unnatural parity singlet states, the coefficient is proportional to $(/+1 / 2)^{-8}$. Moreover, they have shown ${ }^{39}$ that the ratio of the $(/+1 / 2)^{-6}$ and $(/+1 / 2)^{-4}$ coefficients for natural-parity singlet states is not simply $-(5 / 4)$ when the unperturbed wave function is a Hartree-Fock one. Such results generalize to the MøllerPlesset energy of atoms with any number of electrons. ${ }^{39,41}$ Note that the single-term extrapolation formula (2) on the cardinal number $X$ (or $L$, the maximum partial wave number $/ \max$ in a partial wave expansion of the correlation energy) finds its justification in the fact that the leading contribution at secondorder of perturbation theory is proportional to $(/+1 / 2)^{-4}$. Note further that the use of just one term may be accuracy-limiting as the subset of natural singlet-pairs in a MP2 calculation for the $\mathrm{Zn}^{2+}$ ion has been found to contribute only $54.7 \%$ of the total correlation energy. ${ }^{41}$ Although Klopper $^{22}$ has utilized distinct one-term expansions for the singlet and triplet pairs (thus accounting for the $X^{-3}$ and $X^{-5}$ behavior, respectively), we have chosen in our uniform singlet- and triplet-pair extrapolation $\left(\mathrm{USTE}^{27}\right)$ approach not to decompose the total correlation into such contributions. We have done so, first, because it is unnecessary for accurate results; ${ }^{27}$ second, because such a decomposition scheme cannot be implemented for open-shell CCSD calculations ${ }^{26}$ (the wave function is not a spin eigenfunction in practical implementations of CCSD theory) nor is it commonly available in most $\mathrm{CC}$ codes; and third, because such a decomposition is extraneous to MRCI calculations as a single excitation out of the reference space can be counted either as a singlet-pair or as a triplet-pair depending on the spin coupling of the $N-1$ part of the determinant.

In the present work, we follow our previous strategy ${ }^{27}$ by seeking an analytic scheme that extrapolates accurately the correlation energy irrespectively of the diversity of basis set data used for the extrapolations while preserving the correct asymptotic behavior. For practical reasons, it will be dual-level type in the sense that reference calculations for two basis-sets will suffice for the extrapolation; for a one-parameter rule (to our knowledge, the only one available thus far), see ref 20. Since the method is expected to allow reliable extrapolations from energies calculated with any basis sets including those with small $X$ values, one hopes that it will capture the differences between such bases in a realistic manner. In fact, although the angular momentum is not a good quantum number for many-particle systems, and the subsets of wave functions of different angular momentum are difficult to saturate or even balance, it is also true that extrapolations with fairly low / values have shown excellent results ${ }^{22,26,27}$ for small molecules when using cc-type basis sets. ${ }^{11-14}$ Indeed, this has been a major motivation for carrying out the present work.

A final remark goes to the extrapolation of the uncorrelated Hartree-Fock (HF) and complete-active-space-self-consistentfield (CASSCF or simply CAS; uncorrelated in the sense of lacking dynamical correlation) energies. Since it is a topic that lies outside the scope of the present work, we will address the reader to refs 27, 48, 49 (and references therein) for details. Suffice it to say that both extrapolations are geometry-dependent, and hence performed pointwise. Moreover, the use of $\mathrm{HF}$ extrapolated energies plus correlation-scaling/USTE-extrapolated ones $^{50,51}$ has recently shown ${ }^{51,52}$ great promise in obtaining accurate PESs at a low cost, a hybrid approach that will also be of no concern to us here.

The paper is organized as follows. In section 2, we describe the method, while the results are reported and discussed in section 3 . The conclusions are in section 4 .

\section{Method}

The asymptotic limit in eqs 1 and 2 can be imposed by expressing the correlation energy as a rational fraction, which we write in the form

$$
E_{X}^{\mathrm{cor}}=\frac{p_{0}+p_{M} X^{-M}+p_{M+1} X^{-(M+1)} \cdots}{1+q_{N} X^{-N}}
$$

where the $p$ 's and $q$ 's are coefficients, and $M \geq N$; for convenience, we may also think of $X$ as $X+\alpha$. The simplest function of this type is obtained with $M=\infty$, which assumes the form of eqs 1 and 2 if one recalls that for large $X$ values a Taylor expansion in $X^{-1}$ yields $\lim _{X^{-1} \rightarrow 0} 1 /\left(1+q_{N} X^{-N}\right) \sim 1-$ $q_{N} X^{-N}$. Furthermore, if $N=3$, one has $E_{\infty}^{\mathrm{cor}}=p_{0}$, and $A_{3}=$ $-p_{0} q_{3}$. Another variant that will be discussed later consists of limiting the numerator to odd powers, and keeping only the term for $M=5$. In fact, such a continued fraction representation proves to be general and reliable for representing the calculated correlation energies. For example, with $M=\infty$ and the two parameters determined from the results for the two largest cardinal numbers ( 9 and 10) used in ref 45 for the neon atom, one gets $E_{\infty}^{\mathrm{cor}, \mathrm{S}}=-210.64 \mathrm{mE}_{\mathrm{h}}$ and $E_{\infty}^{\mathrm{cor}, \mathrm{T}}=-104.88 \mathrm{mE}_{\mathrm{h}}$ for the singlet-pair and triplet-pair CCSD/VXZ energies, to be compared with $-210.61 \mathrm{mE}_{\mathrm{h}}$ and $-104.87 \mathrm{mE}_{\mathrm{h}}$ from explicitly 
TABLE 1: Ratios $\tau_{53}$ from Valence Shell Correlation Energies Obtained with MP2, CCD and CCSD Calculations ${ }^{a}$

\begin{tabular}{|c|c|c|c|c|c|c|c|c|c|c|c|c|}
\hline \multirow[b]{2}{*}{ system } & \multicolumn{4}{|c|}{ MP2 } & \multicolumn{4}{|c|}{ CCD } & \multicolumn{4}{|c|}{ CCSD } \\
\hline & $\tau_{53^{b}}$ & $\tau_{53}^{D T Q_{c}}$ & $\tau_{53}^{Q 56} d$ & $\bar{\tau}_{53} \pm \Delta \bar{\tau}_{53}{ }^{e}$ & $\tau_{53^{b}}$ & $\tau_{53}^{D T Q_{c}}$ & $\tau_{53}^{Q 56 d}$ & $\bar{\tau}_{53} \pm \Delta \bar{\tau}_{53}{ }^{e}$ & $\tau_{53^{b}}$ & $\tau_{53}^{D T Q_{c}}$ & $\tau_{53}^{Q 56 d}$ & $\bar{\tau}_{53} \pm \Delta \bar{\tau}_{53}{ }^{e}$ \\
\hline $\mathrm{CH}_{2}$ & -1.33 & -1.228513 & -1.319114 & $1.27 \pm 0.05$ & -0.72 & -0.945620 & -0.720395 & $-0.83 \pm 0.11$ & -0.68 & -0.931833 & -0.707320 & $-0.82 \pm 0.11$ \\
\hline $\mathrm{CO}$ & 1 & & & & & & & & & & & \\
\hline $\mathrm{F}_{2}$ & -1.54 & -1.34 & -1.5 & -1.4 & -1.33 & -1.3 & -1.302514 & -1.3 & -1 . & -1 & -1.303 & -1 \\
\hline $\mathrm{H}_{2} \mathrm{O}$ & -1.48 & 8266 & -1.42 & -1.3 & -1.1 & -1 & -1.10 & -1 & -1 & -1 & -1.098821 & 0.06 \\
\hline $\mathrm{HF}$ & -1.5 & -1.347506 & 483362 & $-1.37 \pm 0.06$ & -1.2 & -1.3 & -1.246653 & $-1.28 \pm 0.03$ & & 00331 & -1.245373 & $-1.27 \pm 0.03$ \\
\hline $\mathrm{N}_{2}$ & -1.50 & -1.360448 & -1.498338 & $-1.43 \pm 0.07$ & -1.22 & -1.260942 & -1.190430 & $-1.23 \pm 0.04$ & -1 . & -1.248841 & -1.179869 & $-1.21 \pm 0.03$ \\
\hline $\mathrm{Ne}$ & -1.51 & -1.358759 & -1.564583 & $-1.46 \pm 0.10$ & -1.28 & -1.350284 & -1.357015 & $-1.35 \pm 0.00$ & -1.27 & -1.345554 & -1.348784 & $-1.35 \pm 0.00$ \\
\hline
\end{tabular}

${ }^{a}$ Although the ratios $\tau_{53}^{D T Q}$ and $\tau_{53}^{Q 56}$ are reported in this table and Table 2 with six significant figures as actually employed in the calculations, their average values and associated errors at midpoint (defined such as to embrace both estimates) are quoted for convenience up to two significant figures. ${ }^{b}$ Determined from eq 5 using the values of $A_{3}$ from ref 27. ${ }^{c}$ Determined from a fit of eq 6 to $X=D, T, Q$ energies. ${ }^{d}$ As in $c$ but using up to $X=6$ energies. ${ }^{e}$ Average of $\tau_{53}^{D T Q}$ and $\tau_{53}^{Q 56}$, and error; see the text.

correlated calculations (CCSD-R12) reported in the same paper. Instead, if one chooses $M=5$ with the three parameters determined from calculations for $X=8-10$, one obtains for the singlet-pair energy $E_{\infty}^{\mathrm{cor}, \mathrm{S}}=-210.63 \mathrm{mE}_{\mathrm{h}}$. In fact, the raw nonfitted energies for small- $X$ basis sets consistently show fair to good agreement with the predicted values, thus supporting the reliability of the extrapolation. The rational fraction (3) has indeed been found to be accurate even when unusual asymptotic dependences ( $M$ and $N$ must then be appropriately chosen or left as adjustable parameters) are found as with explicitly correlated energies. ${ }^{53}$ Of course, related forms that preserve the correct asymptotic behavior such as $E_{X}^{\mathrm{cor}}=A \exp \left(-b X^{-3}\right)$ may perform similarly, with the data (partly due to the fact that $X$ is not an exact quantum number) being often unable to discriminate the one that performs best.

The $\mathrm{USTE}^{27}$ scheme has its basis on the simplest threeparameter rule of the above general type in eq 3 . Including the offset parameter $\alpha$, it assumes the form

$$
E_{X}^{\mathrm{cor}}=E_{\infty}^{\mathrm{cor}}+\frac{A_{3}}{(X+\alpha)^{3}}+\frac{A_{5}}{(X+\alpha)^{5}}
$$

where $E_{\infty}^{\mathrm{cor}}, A_{3}$, and $A_{5}$ are parameters to be determined from energies calculated with correlation-consistent basis sets of different cardinal numbers. For a fixed value of $\alpha$, eq 4 is then transformed to the effective two-parameter USTE rule by defining 27

$$
A_{5}=A_{5}^{o}+c A_{3}^{m}
$$

with the parameters $A_{5}^{o}=A_{5}\left(A_{3}=0\right), c$ and $m$ determined from $a b$ initio energies for a variety of systems. For example, from anew ${ }^{27} \mathrm{MRCI}(\mathrm{Q}) / \mathrm{AV} X Z$ calculations for 24 systems, as well as MP2/VXZ, CCD/VXZ, and CCSD/VXZ energies available in the literature ${ }^{22}$ for cardinal numbers ranging from $X=$ $D$ to $X=6$, the following sets of parameters have been obtained with $^{27} \alpha=-3 / 8: A_{5}^{o}=0.003769, c=-1.1784771 \mathrm{E}_{\mathrm{h}}^{-5 / 4}$, and $m=5 / 4$ for $\operatorname{MRCI}(\mathrm{Q}) ; A_{5}^{o}=0.1660699, c=-1.4222512 \mathrm{E}_{\mathrm{h}}^{-1}$, and $m=1$ for the CC family of methods; $A_{5}^{o}=0.0960668, c$ $=-1.582009$ and $m=1$ for MP2. It has further been shown ${ }^{27}$ that both the full correlation in systems studied by MP2 and $\mathrm{CC}[\mathrm{CCD}, \mathrm{CCSD}$, and $\operatorname{CCSD}(\mathrm{T})]$ methods and its dynamical part in $\mathrm{MRCI}(\mathrm{Q})$ calculations $^{52}$ or even correlation energies obtained by correlation energy extrapolation via intrinsic scaling ${ }^{54}$ could be accurately extrapolated to the CBS limit with the USTE rule. Naturally, the above coefficients are likely to vary with the method and the basis set. However, this dependence should not be significant for methods and basis sets
TABLE 2: Ratios $\tau_{53}$ Extracted from MRCI(Q) Calculations

\begin{tabular}{lcccc}
\hline system & $\tau_{53}{ }^{a}$ & $\tau_{53}^{D T Q_{b}}$ & $\tau_{53}^{Q 56 c}$ & $\bar{\tau}_{53}{ }^{d}$ \\
\hline $\mathrm{H}_{2}{ }^{+}$ & -0.28 & -0.182891 & -0.151363 & $-0.17 \pm 0.02$ \\
$\mathrm{H}_{3}{ }^{+}$ & -0.28 & -0.348692 & -0.380301 & $-0.36 \pm 0.02$ \\
$\mathrm{HeH}^{+}$ & -0.46 & -0.805712 & -0.751268 & $-0.78 \pm 0.03$ \\
$\mathrm{He}_{2}{ }^{++}$ & -0.44 & -0.680290 & -0.624268 & $-0.65 \pm 0.03$ \\
$\mathrm{BH}$ & -0.68 & -1.007223 & -0.760193 & $-0.88 \pm 0.12$ \\
$\mathrm{CH}$ & -0.70 & -0.796840 & -0.636825 & $-0.72 \pm 0.08$ \\
$\mathrm{NH}$ & -0.82 & -0.895582 & -0.780484 & $-0.84 \pm 0.06$ \\
$\mathrm{OH}$ & -0.97 & -1.137639 & -1.056519 & $-1.10 \pm 0.04$ \\
$\mathrm{HF}$ & -1.08 & -1.305956 & -1.243364 & $-1.28 \pm 0.03$ \\
$\mathrm{SH}$ & -0.91 & -0.803153 & -0.879484 & $-0.84 \pm 0.04$ \\
$\mathrm{H}_{2} \mathrm{O}$ & & -1.651679 & -1.655947 & $-1.65 \pm 0.00$ \\
$\mathrm{~B}_{2}$ & -0.56 & -0.899805 & -0.517532 & $-0.71 \pm 0.19$ \\
$\mathrm{C}_{2}$ & -0.89 & -1.050512 & -0.843869 & $-0.95 \pm 0.10$ \\
$\mathrm{~N}_{2}$ & -1.06 & -1.135105 & -1.041680 & $-1.09 \pm 0.05$ \\
$\mathrm{O}_{2}$ & -1.16 & -1.195966 & -1.147989 & $-1.17 \pm 0.02$ \\
$\mathrm{~F}_{2}$ & -1.28 & -1.275911 & -1.270502 & $-1.27 \pm 0.00$ \\
$\mathrm{BN}$ & -0.93 & -1.102373 & -0.922511 & $-1.01 \pm 0.09$ \\
$\mathrm{BO}$ & -0.99 & -1.265508 & -1.136348 & $-1.20 \pm 0.07$ \\
$\mathrm{CN}$ & -1.05 & -1.095757 & -0.971588 & $-1.03 \pm 0.07$ \\
$\mathrm{CO}$ & -1.08 & -1.220184 & -1.144859 & $-1.18 \pm 0.04$
\end{tabular}

${ }^{a}$ Determined from eq 5 using the values of $A_{3}$ reported in ref 27. ${ }^{b}$ Determined from a fit of eq 6 to $X=D, T, Q$ energies. ${ }^{c}$ As in $a$ but using up to $X=6\left(X=5\right.$ in the case of $\left.\mathrm{H}_{2} \mathrm{O}\right)$ energies. ${ }^{d}$ Average of $\tau_{53}^{D T Q}$ and $\tau_{53}^{Q 56}$, and error. See footnote $a$ of Table 1 .

that belong to related families, and we have even explored its extendibility ("universality") by showing that accurate results could be obtained for systems not belonging to the calibrating set. ${ }^{49,52}$

The USTE rule may also assume the form

$$
E_{X}^{\mathrm{cor}}=E_{\infty}^{\mathrm{cor}}+\frac{A_{3}}{(X+\alpha)^{3}}\left[1+\frac{\tau_{53}}{(X+\alpha)^{2}}\right]
$$

with the ratio $\tau_{53}$ defined by

$$
\tau_{53}=\frac{A_{5}^{o}}{A_{3}}+c A_{3}^{m-1}
$$

Having the correct asymptotic behavior, one expects eq 4 or eq 6 to be among the best three-parameter models to accurately fit the data, and hence allow a reliable estimate of the ratio $\tau_{53}=$ $A_{5} / A_{3}$ to be extracted from the reported correlation energies. The values of $\tau_{53}$ obtained in this way are reported in Table 1 for seven systems calculated by the MP2, CCD, and CCSD methods with $\mathrm{VXZ}(X=D, T, Q, 5,6)$ basis sets, while Table 2 gathers the results for twenty systems calculated by the MRCI- 
(Q)/AVXZ removed method for the same cardinal numbers. Also shown for comparison are the values obtained from eq 5 using the rounded parameters from Table 1 (second entries) of ref 27. Two observations can be made. First, in the limit $A_{3} \rightarrow 0$ [this coefficient vanishes for pure triplet-pair interactions such as in $\mathrm{He}\left({ }^{3} P_{u}\right)$ or $\left.\mathrm{H}_{3}{ }^{+}\left({ }^{3} \Sigma_{u}^{+}\right)\right]$, numerical problems may arise when determining $E_{\infty}^{\mathrm{cor}}$ and $A_{3}$ due to the division by $A_{3}$ in eq 7 . This can be overcome simply by adding a small constant (say $\delta=10^{-16}$ ) to the denominator of eq 7 . The second observation is of physical nature and concerns the ratio $\tau_{53}$ itself, which assumes the value ${ }^{27}$ of -0.75 for natural-parity singlet states in the $1 / Z$ expansion for various states of the He isoelectronic series [using the standard notation, it corresponds to the ratio ${ }^{39}$ of the $(\zeta+1 / 2)^{-6}$ and $(\zeta+1 / 2)^{-4}$ terms, $\left.a_{2} / a_{1}=-5 / 4\right]$. Note that such a ratio may vary slightly if instead of the wave function for the bare-nuclear Hamiltonian one uses a HF wave function, ${ }^{39}$ as it has actually been shown in MP2 studies of symmetryadapted pairs. ${ }^{41}$ Similarly, it may assume distinct values (unknown thus far) for other levels of theory. ${ }^{39}$

We should observe at this point that the ratio $\tau_{53}$ involves not only the $(l+1 / 2)^{-6}$ term arising from natural parity singlet states but also the leading contribution with the same dependence arising for triplet states. Thus, all electron pairs are essentially involved. Equation 7 with $m=1$ gives for this ratio $\tau_{53}=$ $A_{5}^{o} / A_{3}+c$, thus predicting $\tau_{53}$ to depend both on the value of the intersection at the origin and slope $c$ in eq 5. For $A_{3}=1$, one gets the values of -1.18 for the $\operatorname{MRCI}(\mathrm{Q})$ energies, -1.26 for CCD or CCSD, and -1.49 for MP2, all differing significantly from -0.75 . If one uses instead the optimum values of $A_{3}$ determined in ref 27 , the results in columns two, six and ten of Table 1 as well as those in column two of Table 2 are obtained. As one could anticipate, except for a few systems with small values of $A_{3}$ (notably $\mathrm{H}_{2}, \mathrm{H}_{3}{ }^{+}, \mathrm{HeH}^{+}$, and $\mathrm{He}_{2}{ }^{++}$; see comment above), the agreement between the USTE values and the ones from the present work is generally good.

In the present work, we conjecture that $\tau_{53}$ is invariant over (most) configuration space of the molecule, and hence generalize the USTE model by constraining the extrapolation to reproduce its best estimate as obtained from the fits via eq 6 . Such a procedure has the merit of alerting for the fact that systems with similar values of $A_{3}$ may somehow have distinct $A_{5}$ coefficients, as shown ${ }^{39}$ for helium-like systems with $A_{3}=0$ where distinct triplet electronic states are found to have different values of $A_{5}$. The simplest approach toward this generalization consists of writing

$$
A_{5}=\eta A_{5}^{o}+c A_{3}^{m}
$$

where $\eta$ is a scaling constant that moves the curve in eq 5 up or down parallel to the original law $\left[A_{5}(0)=A_{5}^{o}\right.$, with $\left.\eta=1\right]$ such as to fit best the system under analysis. This is illustrated in Figure 1 for the MP2 energies, with similar plots (including $\mathrm{He}_{2}$ ) shown elsewhere ${ }^{55}$ for CC [CCD, CCSD, and CCSD(T)] and $\mathrm{CI}[\mathrm{CI}(\mathrm{Q})$ and $\mathrm{MRCI}(\mathrm{Q})]$ energies. Of course, this approach will lead to the original three-parameter rule in eq 3 or eq 4 unless an auxiliary criterion is used to fix the system-dependent $\eta$ parameter. Note that a three-parameter model would require three points per geometry to extrapolate a PES, which would be hopelessly time-consuming in a multidimensional situation. We envisage therefore an effective two-parameter generalized USTE (GUSTE) rule.

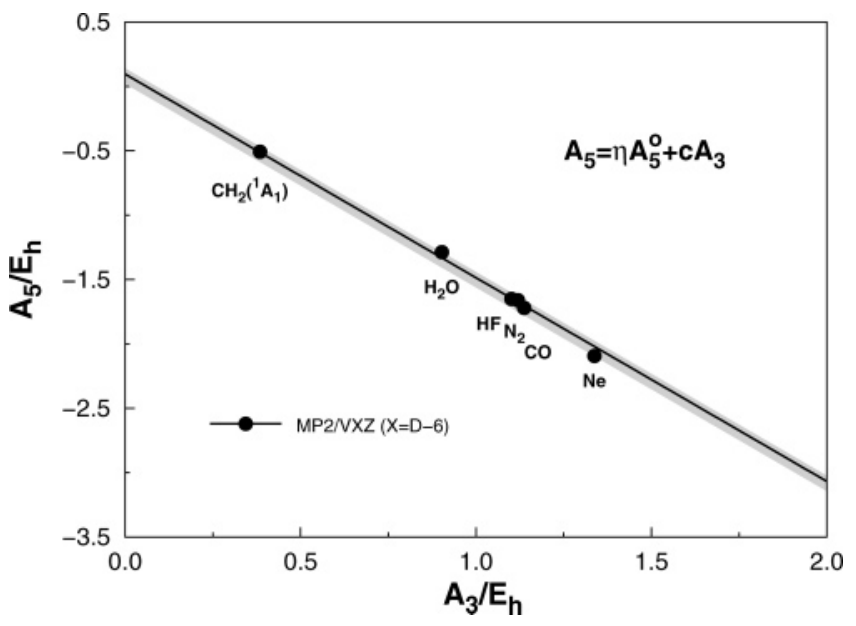

Figure 1. $A_{5}$ vs $A_{3} \operatorname{plot}^{27}$ for MP2 energies calculated with $\mathrm{VXZ}$ correlated consistent orbital basis sets $(X=D, T, Q, 5,6)$. The shadowed area has been obtained by moving the fitted line ${ }^{27,52}$ up and down such as to encompass all calculated points: $0.023 \leq \eta A_{5}^{o} \leq 0.141$. Similar plots for $\mathrm{CC}$ and $\mathrm{CI}(\mathrm{Q})$ energies are shown in ref 55 .

The easiest way to fix $\eta$ is by performing a calculation with the $X=Q$ basis set to obtain $\tau_{53}^{D T Q}$, a variant denoted as GUSTE/DTQ. Thus,

$$
\eta_{D T Q}=A_{3}\left(\tau_{53}^{D T Q}-c A_{3}^{m-1}\right) / A_{5}^{o}
$$

with the label $D T Q$ implying that only calculations with the three smallest cardinal numbers are required. Table 1 gathers the values so obtained. Naturally, $A_{3}$ varies with the cardinal numbers used for the extrapolation, and so does $\eta_{D T Q}$. Clearly, if additional information is available, one may impose the ratio obtained with the three highest affordable cardinal numbers, $\tau_{53}^{Q 56}$. It will then be preferable to avoid the three-point fit to a three-parameter form by using the calculations for the whole set of $X$ values, with larger weights given to increasing $X$ values as in ref 27. The use of the ratios so obtained (still denoted $\tau_{53}^{Q 56}$ ) leads to GUSTE/Q56, while in GUSTE (we reserve the unaltered acronym for this variant of the method) the average ratio $\bar{\tau}_{53}=\left(\tau_{53}^{D T Q}+\tau_{53}^{Q 56}\right) / 2$ is imposed instead. Note that both $\eta_{Q 56}$ and $\eta$ (this refers to GUSTE) vary too with the $X$ values used for the extrapolation.

\section{Results and Discussion}

Table 3 illustrates how the GUSTE method performs when the CCSD/VXZ $(X \leq 10)$ energies of Feller et al. ${ }^{45}$ are utilized for the neon atom. Since the singlet- and triplet-pair energies are treated uniformly by GUSTE, we will consider only their sum for the present analysis. Such raw energies are listed in column two of Table 3, while the extrapolated energies are given in columns six to eight. In turn, columns three to five list the predicted energies for the $X_{2}$ basis sets not involved in the fit. Included for comparison are the results obtained from the $X^{-3}$ law in eq 2 and the $\mathrm{USTE}^{27}$ rule. Since they are nearly coincidental with the GUSTE ones, no further reference to them will be needed. Two other remarks are in order. First, the predicted energies from GUSTE show a tendency to slightly overestimate the unsigned raw energies but never by more than $1 \mathrm{mE}_{\mathrm{h}}$. Conversely, eq 2 tends to underestimate those energies at both $X_{2}$ ends, with deviations up to $5 \mathrm{mE}_{\mathrm{h}}$ or so for $X=T$. Regarding the extrapolated energies, the GUSTE method is seen to overestimate the unsigned CCSD-R $12 \mathrm{~B}^{45}$ explicitly correlated energy by $0.20 \mathrm{mE}_{\mathrm{h}}$, while eq 2 underestimates it by $0.06 \mathrm{mE}_{\mathrm{h}}$. 
TABLE 3: Raw versus CBS Extrapolated Valence Shell CCSD Correlation Energies (in Millihartrees) for the Ground Electronic State of the Neon Atom

\begin{tabular}{|c|c|c|c|c|c|c|c|}
\hline \multirow[b]{2}{*}{$\left(X_{1}, X_{2}\right)^{a}$} & \multicolumn{4}{|c|}{$X_{2}$ energies } & \multicolumn{3}{|c|}{ CBS extrapolated } \\
\hline & $\operatorname{raw}^{b}$ & eq $2^{c}$ & USTE & GUSTE $^{d}$ & eq $2^{c}$ & USTE & GUSTE $^{d}$ \\
\hline$(D, T)$ & -266.34 & -261.11 & -267.33 & -267.30 & & & \\
\hline$(T, Q)$ & -294.68 & -294.80 & -295.35 & -295.35 & -312.03 & -315.24 & -315.22 \\
\hline$(Q, 5)$ & -305.49 & -305.49 & -305.49 & -305.49 & -315.53 & -316.36 & -316.35 \\
\hline$(5,6)$ & -309.90 & -309.90 & -309.90 & -309.90 & -315.42 & -315.68 & -315.68 \\
\hline$(6,7)$ & -312.15 & -312.04 & -312.10 & -312.10 & -315.70 & -315.81 & -315.81 \\
\hline$(7,8)$ & -313.36 & -313.20 & -313.32 & -313.31 & -315.67 & -315.72 & -315.72 \\
\hline$(8,9)$ & -314.02 & -313.89 & -314.04 & -314.04 & -315.50 & -315.52 & -315.52 \\
\hline$(9,10)$ & -314.43 & -314.32 & -314.49 & -314.49 & -315.48 & -315.49 & -315.49 \\
\hline CCSD-R12B & -315.48 & & & & & & \\
\hline $\mathrm{rmsd}^{e}$ & & 1.85 & 0.40 & 0.40 & 1.31 & 0.35 & 0.35 \\
\hline
\end{tabular}

${ }^{a}\left(X_{1}, X_{2}\right)$ denotes the cardinal number pair used for CBS extrapolation. ${ }^{b}$ From ref $45 .{ }^{c}$ Using the offset parameter $\alpha=-3 / 8 .{ }^{d}$ Instead of $\tau_{53}^{D T Q}$, the ratio $\tau_{53}^{T Q 5}=-1.655690$ has been used since the energy for $X=D$ is unavailable, ${ }^{45} \tau_{53}^{Q 56}=-1.260341 .{ }^{e}$ Root mean squared deviation with respect to CCSD-R12B.

TABLE 4: CBS Extrapolated Valence Shell MP2, CCD and CCSD Correlation Energies (in Millihartrees) for the Ground Electronic States of $\mathrm{CH}_{2}, \mathrm{CO}, \mathrm{F}_{2}, \mathrm{H}_{2} \mathrm{O}, \mathrm{N}_{2}$, and $\mathrm{Ne}$

\begin{tabular}{|c|c|c|c|c|c|c|c|c|c|c|c|c|c|}
\hline \multirow[b]{2}{*}{ system } & \multirow[b]{2}{*}{$\left(X_{1}, X_{2}\right)^{a}$} & \multicolumn{4}{|c|}{ MP2 } & \multicolumn{4}{|c|}{ CCD } & \multicolumn{4}{|c|}{ CCSD } \\
\hline & & & eq $2^{c}$ & $\mathrm{USTE}^{d}$ & GUSTE $^{e}$ & & eq $2^{c}$ & $\mathrm{USTE}^{d}$ & GUSTE $^{e}$ & & eq $2^{c}$ & $\mathrm{USTE}^{d}$ & GUSTE $^{e}$ \\
\hline $\mathrm{CH}_{2}$ & $\begin{array}{l}(D, T) \\
(T, Q) \\
(Q, 5) \\
(5,6)\end{array}$ & & $\begin{array}{l}-147.5 \\
-153.9 \\
-155.2 \\
-155.4\end{array}$ & $\begin{array}{l}-156.8 \\
-155.0 \\
-155.2 \\
-155.5\end{array}$ & $\begin{array}{l}-155.6 \pm 0.7^{f} \\
-155.0 \pm 0.1 \\
-155.5 \pm 0.0 \\
-155.5 \pm 0.0\end{array}$ & & & $\begin{array}{l}-173.7 \\
-175.4 \\
-174.8 \\
-174.8\end{array}$ & $\begin{array}{l}-174.7 \pm 0.7^{g} \\
-175.4 \pm 0.1 \\
-174.8 \pm 0.0 \\
-174.8 \pm 0.0\end{array}$ & & & & $\begin{array}{l}5.6 \pm 0.7^{f} \\
6.3 \pm 0.1 \\
5.8 \pm 0.0 \\
5.7 \pm 0.0\end{array}$ \\
\hline $\mathrm{CO}$ & $\begin{array}{l}(D, T) \\
(T, Q) \\
(Q, 5) \\
(5,6)\end{array}$ & & $\begin{array}{l}-376.6 \\
-396.9 \\
-401.7 \\
-402.6\end{array}$ & $\begin{array}{l}-406.9 \\
-400.5 \\
-402.7 \\
-403.0\end{array}$ & $\begin{array}{l}-403.6 \pm 3.7^{f} \\
-400.4 \pm 0.2 \\
-402.7 \pm 0.1 \\
-402.9 \pm 0.0\end{array}$ & & $\begin{array}{l}-373.9 \\
-390.5 \\
-391.9 \\
-392.2\end{array}$ & $\begin{array}{l}-390.4 \\
-392.9 \\
-392.5 \\
-392.2\end{array}$ & $\begin{array}{l}-392.0 \pm 1.0^{g} \\
-393.0 \pm 0.1 \\
-392.5 \pm 0.0 \\
-392.2 \pm 0.0\end{array}$ & & & $\begin{array}{l}-394.4 \\
-396.7 \\
-396.4 \\
-395.9\end{array}$ & $\begin{array}{l}-395.7 \pm 1.1^{f} \\
-396.7 \pm 0.1 \\
-396.4 \pm 0.0 \\
-395.9 \pm 0.0\end{array}$ \\
\hline $\mathrm{F}_{2}$ & $\begin{array}{l}(D, T) \\
(T, Q) \\
(Q, 5) \\
(5,6)\end{array}$ & $\begin{array}{l}-599.1 \\
-608.5 \\
-609.9\end{array}$ & $\begin{array}{l}-562.4 \\
-598.3 \\
-608.1 \\
-609.5\end{array}$ & $\begin{array}{l}-624.4 \\
-605.2 \\
-610.0 \\
-610.2\end{array}$ & $\begin{array}{l}-612.0 \pm 8.9^{f} \\
-604.6 \pm 0.5 \\
-609.8 \pm 0.1 \\
-610.1 \pm 0.0\end{array}$ & $\begin{array}{l}-592.0 \\
-596.3 \\
-596.0\end{array}$ & $\begin{array}{l}-559.1 \\
-591.1 \\
-596.1 \\
-595.8\end{array}$ & $\begin{array}{l}-597.7 \\
-596.4 \\
-597.5 \\
-596.2\end{array}$ & $\begin{array}{l}-596.1 \pm 0.2^{g} \\
-596.2 \pm 0.0 \\
-597.4 \pm 0.0 \\
-596.2 \pm 0.0\end{array}$ & & $\begin{array}{l}-564.3 \\
-596.5 \\
-601.3 \\
-601.2\end{array}$ & $\begin{array}{l}-603.1 \\
-601.7 \\
-602.7 \\
-601.6\end{array}$ & $\begin{array}{l}-601.6 \pm 0.0^{f} \\
-601.6 \pm 0.0 \\
-602.6 \pm 0.0 \\
-601.6 \pm 0.0\end{array}$ \\
\hline $\mathrm{H}_{2} \mathrm{O}$ & $\begin{array}{l}(D, T) \\
(T, Q) \\
(Q, 5) \\
(5,6)\end{array}$ & $\begin{array}{l}-296.4 \\
-299.8 \\
-300.0\end{array}$ & $\begin{array}{l}-280.1 \\
-295.8 \\
-299.6 \\
-299.8\end{array}$ & $\begin{array}{l}-306.0 \\
-298.8 \\
-300.4 \\
-300.1\end{array}$ & $\begin{array}{l}-300.5 \pm 2.3^{f} \\
-298.5 \pm 0.2 \\
-300.3 \pm 0.0 \\
-300.1 \pm 0.0\end{array}$ & & $\begin{array}{l}-283.7 \\
-296.1 \\
-297.1 \\
-296.8\end{array}$ & $\begin{array}{l}-297.2 \\
-298.1 \\
-297.8 \\
-297.0\end{array}$ & $\begin{array}{l}-296.7 \pm 1.3^{g} \\
-298.0 \pm 0.1 \\
-297.6 \pm 0.0 \\
-297.0 \pm 0.0\end{array}$ & & & $\begin{array}{l}-298.3 \\
-299.3 \\
-298.8 \\
-298.2\end{array}$ & \\
\hline $\mathrm{HF}$ & $\begin{array}{l}(D, T) \\
(T, Q) \\
(Q, 5) \\
(5,6)\end{array}$ & & $\begin{array}{l}-293.5 \\
-313.3 \\
-318.1 \\
-318.6\end{array}$ & $\begin{array}{l}-324.8 \\
-316.9 \\
-319.1 \\
-318.9\end{array}$ & $\begin{array}{l}-317.3 \pm 2.7^{g} \\
-316.5 \pm 0.2 \\
-319.0 \pm 0.0 \\
-318.9 \pm 0.0\end{array}$ & $\begin{array}{l}-311.5 \\
-313.2 \\
-312.8\end{array}$ & $\begin{array}{l}-293.5 \\
-310.8 \\
-313.0 \\
-312.7\end{array}$ & $\begin{array}{l}-311.1 \\
-313.5 \\
-313.6 \\
-312.8\end{array}$ & $\begin{array}{l}-312.6 \pm 1.0^{g} \\
-313.5 \pm 0.1 \\
-313.7 \pm 0.0 \\
-312.9 \pm 0.0\end{array}$ & & & $\begin{array}{l}-312.3 \\
-314.7 \\
-315.0 \\
-314.1\end{array}$ & $\begin{array}{l}-313.9 \pm 1.0^{f} \\
-314.8 \pm 0.1 \\
-315.0 \pm 0.0 \\
-314.2 \pm 0.0\end{array}$ \\
\hline $\mathrm{N}_{2}$ & $\begin{array}{l}(D, T) \\
(T, Q) \\
(Q, 5) \\
(5,6)\end{array}$ & $\begin{array}{l}-414.9 \\
-419.2 \\
-420.9\end{array}$ & $\begin{array}{l}-394.8 \\
-414.3 \\
-418.8 \\
-419.8\end{array}$ & $\begin{array}{l}-424.6 \\
-417.8 \\
-419.7 \\
-420.1\end{array}$ & $\begin{array}{l}-420.6 \pm 3.5^{g} \\
-417.6 \pm 0.2 \\
-419.7 \pm 0.1 \\
-420.1 \pm 0.0\end{array}$ & $\begin{array}{l}-403.0 \\
-404.1 \\
-403.9\end{array}$ & $\begin{array}{l}-387.6 \\
-402.5 \\
-404.0 \\
-403.8\end{array}$ & $\begin{array}{l}-403.5 \\
-404.8 \\
-404.5 \\
-403.9\end{array}$ & $\begin{array}{l}-403.8 \pm 1.0^{g} \\
-404.8 \pm 0.1 \\
-404.5 \pm 0.0 \\
-403.9 \pm 0.0\end{array}$ & $\begin{array}{l}-406.8 \\
-407.8 \\
-407.6\end{array}$ & $\begin{array}{l}-391.5 \\
-406.2 \\
-407.7 \\
-407.5\end{array}$ & $\begin{array}{l}-407.3 \\
-408.4 \\
-408.2 \\
-407.6\end{array}$ & $\begin{array}{l}-407.5 \pm 1.0^{f} \\
-408.4 \pm 0.1 \\
-408.2 \pm 0.0 \\
-407.6 \pm 0.0\end{array}$ \\
\hline $\mathrm{Ne}$ & $\begin{array}{l}(D, T) \\
(T, Q) \\
(Q, 5) \\
(5,6)\end{array}$ & $\begin{array}{l}-312.1 \\
-318.2 \\
-319.0\end{array}$ & $\begin{array}{l}-288.8 \\
-311.5 \\
-317.9 \\
-318.8\end{array}$ & $\begin{array}{l}-324.6 \\
-315.7 \\
-319.1 \\
-319.2\end{array}$ & $\begin{array}{l}-321.0 \pm 6.8^{g} \\
-315.6 \pm 0.4 \\
-319.0 \pm 0.1 \\
-319.2 \pm 0.0\end{array}$ & $\begin{array}{l}-312.0 \\
-314.9 \\
-314.8\end{array}$ & $\begin{array}{l}-289.5 \\
-311.2 \\
-314.7 \\
-314.6\end{array}$ & $\begin{array}{l}-310.9 \\
-314.4 \\
-315.6 \\
-314.9\end{array}$ & $\begin{array}{l}-314.9 \pm 0.2^{f} \\
-314.7 \pm 0.0 \\
-315.6 \pm 0.0 \\
-314.9 \pm 0.0\end{array}$ & $\begin{array}{l}-312.8 \\
-315.8 \\
-315.6\end{array}$ & $\begin{array}{l}-290.3 \\
-312.1 \\
-315.5 \\
-315.4\end{array}$ & $\begin{array}{l}-311.7 \\
-315.3 \\
-316.4 \\
-315.6\end{array}$ & $\begin{array}{l}-315.6 \pm 0.1^{g} \\
-315.6 \pm 0.0 \\
-316.4 \pm 0.0 \\
-315.7 \pm 0.0\end{array}$ \\
\hline
\end{tabular}

${ }^{a}\left(X_{1}, X_{2}\right)$ denotes the cardinal number pair used for CBS extrapolation. ${ }^{b}$ Reference 22 . See this reference for further comparisons. ${ }^{c}$ Using the offset parameter $\alpha=-3 / 8 .{ }^{d}$ Reference $27 .{ }^{e}$ This work, with the error defined such as to encompass the results from GUSTE/DQT and GUSTE/ Q56. ${ }^{f}$ The lower bound for this system is obtained with $\eta_{D T Q}{ }^{g}$ The lower bound for this system is obtained with $\eta_{Q 56 .}$.

Since eq 2 also underestimates the target unsigned energy at $X_{2}=10$, and the CCSD-R12B energy is itself not free from error, it will be impossible to say which estimate is more reliable. However, the rms error in GUSTE is seen to be a factor of 4 smaller than for eq 2 .

We now turn to Tables 4-9which compare the results obtained from the GUSTE method with the USTE ${ }^{27}$ and traditional $X^{-3}$ laws for MP2, CCD, CCSD, and MRCI(Q) energies. As in the previous paragraph, the GUSTE energies provide a substantial improvement relative to the results from eq 2 or GUSTE/DTQ. However, such improvements are relatively small compared with the USTE results, except for the $(D, T)$ extrapolated energy that tends to lie closer to the $(5,6)$ result. A similar pattern is observed for GUSTE/Q56, with the improvements over USTE being now expected for extrapolated energies with larger $X$ values. Relatively small, albeit significant, improvements arise though from GUSTE, but (as in GUSTE/ Q56) at the expense of knowing $\tau_{53}^{Q 56}$ which implies calculations with the largest affordable cardinal numbers. For two electron systems, where exact energies are available, the differences between the GUSTE and USTE energies are predicted to be in the micro- or submicrohartree range, amounting at most to $-3 \mu \mathrm{E}_{\mathrm{h}}$ for $\mathrm{HeH}^{+}$. A favorable case is $\mathrm{He}_{2}{ }^{++}$, 
TABLE 5: Extrapolated MRCI(Q) Dynamical Correlation Energies (in Millihartrees) for the Ground Electronic States of Some Two-Electron Systems ${ }^{a}$

\begin{tabular}{ccccc}
\hline system & $\left(X_{1}, X_{2}\right)^{b}$ & eq $2^{c}$ & $\mathrm{USTE}^{d}$ & $\mathrm{GUSTE}^{e}$ \\
\hline $\mathrm{H}_{2}$ & $(D, T)$ & -22.249 & -22.363 & $-22.310 \pm 0.006^{f}$ \\
& $(T, Q)$ & -22.307 & -22.321 & $-22.315 \pm 0.001$ \\
& $(Q, 5)$ & -22.320 & -22.324 & $-22.322 \pm 0.000$ \\
& $(5,6)$ & -22.307 & -22.308 & $-22.308 \pm 0.000$ \\
$\mathrm{H}_{3}{ }^{+}$ & $(D, T)$ & -13.031 & -13.067 & $-13.165 \pm 0.007^{g}$ \\
& $(T, Q)$ & -13.140 & -13.154 & $-13.159 \pm 0.001$ \\
& $(Q, 5)$ & -13.194 & -13.198 & $-13.199 \pm 0.000$ \\
& $(5,6)$ & -13.161 & -13.162 & $-13.163 \pm 0.000$ \\
$\mathrm{HeH}^{+}$ & $(D, T)$ & -23.295 & -23.428 & $-23.889 \pm 0.032^{f}$ \\
& $(T, Q)$ & -23.836 & -23.880 & $-23.919 \pm 0.003$ \\
& $(Q, 5)$ & -23.890 & -23.902 & $-23.910 \pm 0.001$ \\
& $(5,6)$ & -23.886 & -23.890 & $-23.893 \pm 0.000$ \\
$\mathrm{He}_{2}{ }^{++}$ & $(D, T)$ & -16.614 & -16.853 & $-17.063 \pm 0.027^{f}$ \\
& $(T, Q)$ & -17.025 & -17.064 & $-17.088 \pm 0.003$ \\
& $(Q, 5)$ & -17.079 & -17.089 & $-17.094 \pm 0.001$ \\
& $(5,6)$ & -17.065 & -17.068 & $-17.070 \pm 0.000$
\end{tabular}

${ }^{a}$ Similar to ref 27 , we quote the extrapolated MRCI(Q) energies with microhartree accuracy. The error indicated in the last column of this and the following tables (Table 2 included as well) measures the variation associated with the use of $\tau_{53}^{D T Q}$ and $\tau_{53}^{Q 56}$ which should not be confused with the error relative to the exact nonrelativistic energy (or the true CBS limit for systems with more electrons) that is difficult to obtain for all but few-electron systems (see ref 54). ${ }^{b}\left(X_{1}, X_{2}\right)$ denotes the cardinal number pair used for extrapolation. ${ }^{c}$ Using the offset parameter $\alpha=-3 / 8 .{ }^{d}$ Reference 27. ${ }^{e}$ This work. ${ }^{f}$ The lower bound for this system is obtained with $\eta_{D T Q} .{ }^{g}$ The lower bound for this system is obtained with $\eta_{Q 56}$.

where the total energy (the CAS energy has been taken from our best estimate in ref 27) is predicted to be $-3681.456 \mathrm{mE}_{\mathrm{h}}$, in excellent agreement with Wolniewicz's ${ }^{56}$ result. Note that the requirement of knowing $\tau_{53}^{Q 56}$ should pose no significant limitation for many systems of interest, since our experience suggests that calculations at a single geometry should suffice to calibrate the method. Note especially that the conjecture that $\tau_{53}$ is (approximately) invariant over the molecule configuration space finds support in exploratory calculations for diatomic molecules where the extrapolated energies appear to be predicted reliably from the repulsive wing of the potential curve up to dissociation. In fact, although the number of coupled electron pairs is expected to diminish upon bond breaking, such a fluctuation is likely to be small. Moreover, their number should vary smoothly with separation, suggesting that any small change in the scaling factor used for the extrapolation may largely be irrelevant. Unfortunately, we are not aware of any rigorous study of this problem for a polyatomic molecule.

The only piece of data at our disposal to test the above conjecture refers to the MRCI(Q)/AVXZ energies for the ground electronic states of $\mathrm{H}_{2} \mathrm{O}$ and $\mathrm{OH}$, as the latter can be a dissociation product of the former (for a recent accurate $a b$ initio study on the water molecule that also addresses CBS extrapolation, see ref 57). Although the calculated $\tau_{53}$ values seem to bear little relation to each other, we alert for the fact that they have been estimated using different sets of cardinal numbers. In an attempt to assess the performance of the GUSTE method for $\mathrm{H}_{2} \mathrm{O}$, we have therefore examined in some detail the dependence in $X$ of the dynamical correlation energy. The atypical pattern illustrated in panel (a) of Figure 2 may be rationalized as due to the fact that the basis is saturated faster for $\mathrm{OH}$ than $\mathrm{H}_{2} \mathrm{O}$. This can be understood by considering the dynamical correlation per electron. For $\mathrm{H}_{2} \mathrm{O}$, we will first divide the total dynamical correlation by 10 electrons (valence plus
TABLE 6: Extrapolated MRCI(Q) Valence Dynamical Correlation Energies (in Millihartrees) for the Ground Electronic States of Some Hydrides (For the Water Molecule, the Core-Correlation Energy Has Also Been Included)

\begin{tabular}{|c|c|c|c|c|}
\hline system & $\left(X_{1}, X_{2}\right)^{a}$ & eq $2^{b}$ & $\mathrm{USTE}^{c}$ & $\mathrm{GUSTE}^{d}$ \\
\hline $\mathrm{BH}$ & $\begin{array}{l}(D, T) \\
(T, Q) \\
(Q, 5) \\
(5,6)\end{array}$ & $\begin{array}{l}-49.398 \\
-50.621 \\
-50.585 \\
-50.547\end{array}$ & $\begin{array}{l}-49.696 \\
-50.721 \\
-50.607 \\
-50.554\end{array}$ & $\begin{array}{l}-50.527 \pm 0.265^{e} \\
-50.788 \pm 0.028 \\
-50.621 \pm 0.006 \\
-50.558 \pm 0.002\end{array}$ \\
\hline $\mathrm{CH}$ & $\begin{array}{l}(D, T) \\
(T, Q) \\
(Q, 5) \\
(5,6)\end{array}$ & $\begin{array}{l}-100.353 \\
-102.212 \\
-102.265 \\
-102.191\end{array}$ & $\begin{array}{l}-102.160 \\
-102.475 \\
-102.325 \\
-102.209\end{array}$ & $\begin{array}{l}-102.189 \pm 0.304^{e} \\
-102.475 \pm 0.034 \\
-102.326 \pm 0.007 \\
-102.210 \pm 0.002\end{array}$ \\
\hline $\mathrm{NH}$ & $\begin{array}{l}(D, T) \\
(T, Q) \\
(Q, 5) \\
(5,6)\end{array}$ & $\begin{array}{l}-153.701 \\
-157.205 \\
-157.484 \\
-157.338\end{array}$ & $\begin{array}{l}-157.232 \\
-157.717 \\
-157.606 \\
-157.374\end{array}$ & $\begin{array}{l}-157.345 \pm 0.403^{e} \\
-157.723 \pm 0.042 \\
-157.608 \pm 0.009 \\
-157.375 \pm 0.003\end{array}$ \\
\hline $\mathrm{OH}$ & $\begin{array}{l}(D, T) \\
(T, Q) \\
(Q, 5) \\
(5,6)\end{array}$ & $\begin{array}{l}-217.260 \\
-225.550 \\
-226.361 \\
-226.208\end{array}$ & $\begin{array}{l}-223.989 \\
-226.643 \\
-226.629 \\
-226.289\end{array}$ & $\begin{array}{l}-226.198 \pm 0.655^{e} \\
-226.817 \pm 0.059 \\
-226.667 \pm 0.013 \\
-226.302 \pm 0.004\end{array}$ \\
\hline $\mathrm{FH}$ & $\begin{array}{l}(D, T) \\
(T, Q) \\
(Q, 5) \\
(5,6)\end{array}$ & $\begin{array}{l}-276.841 \\
-291.304 \\
-292.706 \\
-292.704\end{array}$ & $\begin{array}{l}-286.828 \\
-293.116 \\
-293.152 \\
-292.844\end{array}$ & $\begin{array}{l}-292.672 \pm 0.916^{e} \\
-293.546 \pm 0.072 \\
-293.244 \pm 0.015 \\
-292.874 \pm 0.005\end{array}$ \\
\hline $\mathrm{SH}$ & $\begin{array}{l}(D, T) \\
(T, Q) \\
(Q, 5) \\
(5,6)\end{array}$ & $\begin{array}{l}-186.417 \\
-190.617 \\
-191.453 \\
-191.568\end{array}$ & $\begin{array}{l}-192.449 \\
-191.387 \\
-191.651 \\
-191.633\end{array}$ & $\begin{array}{l}-191.662 \pm 0.384^{e} \\
-191.327 \pm 0.038 \\
-191.635 \pm 0.009 \\
-191.628 \pm 0.003\end{array}$ \\
\hline $\mathrm{H}_{2} \mathrm{O}$ & $\begin{array}{l}(D, T) \\
(T, Q) \\
(Q, 5)\end{array}$ & $\begin{array}{l}-261.355 \\
-293.458 \\
-297.486\end{array}$ & $\begin{array}{l}-272.809 \\
-296.888 \\
-298.399\end{array}$ & $\begin{array}{l}-298.786 \pm 0.190^{f} \\
-298.606 \pm 0.010 \\
-298.755 \pm 0.002\end{array}$ \\
\hline
\end{tabular}

${ }^{a}\left(X_{1}, X_{2}\right)$ denotes the cardinal number pair used for extrapolation. ${ }^{b}$ Using the offset parameter $\alpha=-3 / 8 .{ }^{c}$ Reference 27. ${ }^{d}$ This work. ${ }^{e}$ The lower bound for this system is obtained with $\eta_{D T Q}{ }^{f}$ The lower bound for this system is obtained with $\eta_{Q 56}$.

core) as the calculations on this molecule included corecorrelation effects: the lowest-energy molecular orbital $(\sim 1 \mathrm{~s}$ orbital of oxygen) was optimized in the CASSCF calculations, but unlike active orbitals that change occupations it remained doubly occupied in all reference determinants defining the CASSCF and MRCI wave functions. The calculated dynamical correlation energy per electron so obtained varies from -18.24 $\mathrm{mE}_{\mathrm{h}}$ for $X=D$ to $-24.26 \mathrm{mE}_{\mathrm{h}}$ for $X=T$ and $-28.62 \mathrm{mE}_{\mathrm{h}}$ for $X=5$. However, not all the core-valence correlation has been recovered due to having kept closed the 1s orbitals in the CASSCF and MRCI calculations. Thus, one may consider that the calculated dynamical correlation is essentially of the valence type, yielding for the dynamical correlation energy per valence electron the values of $-22.79,-30.33$, and $-35.78 \mathrm{mE}_{\mathrm{h}}$ in the above order. For $\mathrm{OH}$ (and all other systems listed in Tables 6-8), core correlation has been ignored, with the valence dynamical correlation per electron varying from $-22.95 \mathrm{mE}_{\mathrm{h}}$ for $X=D$ to $-29.11 \mathrm{mE}_{\mathrm{h}}$ for $X=T$ and $-31.71 \mathrm{mE}_{\mathrm{h}}$ for $X=$ 5 . As could be expected, ${ }^{8}$ this is generally smaller for the openshell hydroxyl radical than for the closed-shell water molecule, where all electrons are paired. Another indicator that the basis is saturated faster for $\mathrm{OH}$ than $\mathrm{H}_{2} \mathrm{O}$ is the corresponding fraction of recovered correlation energy for $X=D, T$, and 5: 71, 90, and $98 \%$ of the $(5,6)$ valence dynamical correlation energy for $\mathrm{OH} ; 61,81$, and $96 \%$ of the $(Q, 5)$ extrapolated (mostly valence) dynamical correlation for $\mathrm{H}_{2} \mathrm{O}$. This may even be inferred from the slightly distinct slopes displayed for $X \geq 4$ by the curves in Figures 2 and 3 for the CBS extrapolations in $\mathrm{H}_{2} \mathrm{O}$ and $\mathrm{OH}$, 
TABLE 7: Extrapolated MRCI(Q) Valence Dynamical Correlation Energies (in Millihartrees) for the Ground Electronic States of First-Row Homonuclear Diatomics

\begin{tabular}{ccccc}
\hline system & $\left(X_{1}, X_{2}\right)^{a}$ & eq $2^{b}$ & USTE $^{c}$ & GUSTE $^{d}$ \\
\hline $\mathrm{B}_{2}$ & $(D, T)$ & -88.242 & -89.971 & $-89.995 \pm 0.707^{e}$ \\
& $(T, Q)$ & -90.440 & -90.712 & $-90.705 \pm 0.083$ \\
& $(Q, 5)$ & -90.212 & -90.266 & $-90.268 \pm 0.016$ \\
& $(5,6)$ & -90.092 & -90.108 & $-90.109 \pm 0.005$ \\
$\mathrm{C}_{2}$ & $(D, T)$ & -160.492 & -165.857 & $-166.374 \pm 1.128^{e}$ \\
& $(T, Q)$ & -166.614 & -167.447 & $-167.476 \pm 0.114$ \\
& $(Q, 5)$ & -166.718 & -166.904 & $-166.914 \pm 0.024$ \\
& $(5,6)$ & -166.554 & -166.610 & $-166.614 \pm 0.007$ \\
$\mathrm{~N}_{2}$ & $(D, T)$ & -266.477 & -278.039 & $-278.824 \pm 1.043^{e}$ \\
& $(T, Q)$ & -278.057 & -279.774 & $-279.815 \pm 0.095$ \\
& $(Q, 5)$ & -279.149 & -279.561 & $-279.572 \pm 0.020$ \\
& $(5,6)$ & -278.914 & -279.038 & $-279.044 \pm 0.006$ \\
$\mathrm{O}_{2}$ & $(D, T)$ & -404.121 & -423.215 & $-423.456 \pm 0.841^{e}$ \\
& $(T, Q)$ & -421.529 & -424.245 & $-424.244 \pm 0.071$ \\
& $(Q, 5)$ & -423.502 & -424.163 & $-424.164 \pm 0.015$ \\
& $(5,6)$ & -423.397 & -423.601 & $-423.605 \pm 0.005$ \\
$\mathrm{~F}_{2}$ & $(D, T)$ & -506.577 & -537.208 & $-536.722 \pm 0.151^{e}$ \\
& $(T, Q)$ & -532.692 & -536.891 & $-536.861 \pm 0.012$ \\
& $(Q, 5)$ & -536.420 & -537.478 & $-537.464 \pm 0.003$ \\
& $(5,6)$ & -536.431 & -536.761 & $-536.762 \pm 0.001$
\end{tabular}

${ }^{a}\left(X_{1}, X_{2}\right)$ denotes the cardinal number pair used for extrapolation. ${ }^{b}$ Using the offset parameter $\alpha=-3 / 8 .{ }^{c}$ Reference 27. ${ }^{d}$ This work.

${ }^{e}$ The lower bound for this system is obtained with $\eta_{D T Q}$.

TABLE 8: Extrapolated MRCI(Q) Valence Dynamical Correlation Energies (in Millihartrees) for the Ground Electronic States of BN, BO, CN and CO

\begin{tabular}{ccccc}
\hline system & $\left(X_{1}, X_{2}\right)^{a)}$ & eq $2^{b}$ & USTE $^{c}$ & GUSTE $^{d}$ \\
\hline BN & $(D, T)$ & -178.383 & -184.495 & $-185.592 \pm 1.186^{e}$ \\
& $(T, Q)$ & -185.696 & -186.671 & $-186.749 \pm 0.115$ \\
& $(Q, 5)$ & -186.021 & -186.244 & $-186.264 \pm 0.024$ \\
& $(5,6)$ & -185.775 & -185.840 & $-185.848 \pm 0.007$ \\
BO & $(D, T)$ & -215.559 & -224.710 & $-228.659 \pm 1.544^{e}$ \\
& $(T, Q)$ & -228.267 & -229.878 & $-230.167 \pm 0.132$ \\
& $(Q, 5)$ & -229.087 & -229.465 & $-229.529 \pm 0.027$ \\
& $(5,6)$ & -228.967 & -229.083 & $-229.104 \pm 0.008$ \\
CN & $(D, T)$ & -219.324 & -227.470 & $-228.322 \pm 1.016^{e}$ \\
& $(T, Q)$ & -228.002 & -229.240 & $-229.294 \pm 0.096$ \\
& $(Q, 5)$ & -228.639 & -228.930 & $-228.945 \pm 0.021$ \\
& $(5,6)$ & -228.426 & -228.513 & $-228.519 \pm 0.006$ \\
CO & $(D, T)$ & -266.510 & -277.805 & $-280.941 \pm 0.987$ \\
& $(T, Q)$ & -279.831 & -281.660 & $-281.879 \pm 0.084$ \\
& $(Q, 5)$ & -281.147 & -281.593 & $-281.641 \pm 0.018$ \\
& $(5,6)$ & -281.015 & -281.152 & $-281.169 \pm 0.005$
\end{tabular}

${ }^{a}\left(X_{1}, X_{2}\right)$ denotes the cardinal number pair used for extrapolation. ${ }^{b}$ Using the offset parameter $\alpha=-3 / 8 .{ }^{c}$ Reference 27. ${ }^{d}$ This work. ${ }^{e}$ The lower bound for this system is obtained with $\eta_{D T Q}$.

respectively. Thus, a balanced level of description is obtained faster for $\mathrm{OH}$ than $\mathrm{H}_{2} \mathrm{O}$, which may explain the small plateau in plot (a) of Figure 2. We should note that removing the $X=$ $D$ or $T$ energies from the fit will not change the above pattern. A more typical trend may, however, be obtained if the AVDZ energy is replaced by $-147 \mathrm{mE}_{\mathrm{h}}$ or so rather than the actually calculated value of $-182.34 \mathrm{mE}_{\mathrm{h}}$; see panel (b) of Figure 2. Using the former, the following extrapolated correlation energies are obtained for the water molecule (format as in Table 6): $-298.782 \pm 2.123(D, T),-296.988 \pm 0.155(T, Q)$, and $-298.405 \pm 0.035(Q, 5)$. They correspond to $\tau_{53}^{D T Q}=1.21$ and $\tau_{53}^{Q 56}=1.30$, in somewhat closer agreement with $\tau_{53}^{Q 56}(\mathrm{OH})$. The improvement is striking for the $(D, T)$ extrapolation, while remaining good for $(Q, 5)$ when comparing with USTE. Fur-
TABLE 9: A Comparison of the Calculated GUSTE/DTQ and GUSTE/DD + T Extrapolated CCSD Correlation Energies (in Millihartrees) and Other Parameters for the Ground Electronic States of $\mathrm{CH}_{2}, \mathrm{CO}, \mathrm{F}_{2}, \mathrm{H}_{2} \mathrm{O}, \mathrm{HF}, \mathrm{N}_{2}$, and $\mathrm{Ne}$

\begin{tabular}{|c|c|c|c|c|c|}
\hline system & $\left(X_{1}, X_{2}\right)^{a}$ & GUSTE/DTQ & $\eta_{D T Q}^{b}$ & GUSTE $/ D D_{+} T$ & $\tau_{53}^{D D+T_{c}}$ \\
\hline $\mathrm{CH}_{2}$ & $\begin{array}{l}(D, T) \\
(T, Q) \\
(Q, 5) \\
(5,6)\end{array}$ & $\begin{array}{l}-176.4 \\
-176.4 \\
-175.8 \\
-175.7\end{array}$ & $\begin{array}{l}0.751 \\
0.751 \\
0.660 \\
0.631\end{array}$ & $\begin{array}{l}-175.9 \\
-176.3 \\
-175.7 \\
-175.7\end{array}$ & $\begin{array}{l}-0.864 \\
-0.886 \\
-0.811 \\
-0.784\end{array}$ \\
\hline $\mathrm{CO}$ & $\begin{array}{l}(D, T) \\
(T, Q) \\
(Q, 5) \\
(5,6)\end{array}$ & $\begin{array}{l}-396.8 \\
-396.8 \\
-396.5 \\
-395.9\end{array}$ & $\begin{array}{l}0.694 \\
0.694 \\
0.678 \\
0.630\end{array}$ & $\begin{array}{l}-386.7 \\
-396.2 \\
-396.3 \\
-395.8\end{array}$ & $\begin{array}{l}-0.864 \\
-1.013 \\
-1.017 \\
-0.991\end{array}$ \\
\hline $\mathrm{F}_{2}$ & $\begin{array}{l}(D, T) \\
(T, Q) \\
(Q, 5) \\
(5,6)\end{array}$ & $\begin{array}{l}-601.6 \\
-601.6 \\
-602.6 \\
-601.6\end{array}$ & $\begin{array}{l}1.197 \\
1.197 \\
1.236 \\
1.158\end{array}$ & $\begin{array}{l}-581.0 \\
-600.2 \\
-602.3 \\
-601.5\end{array}$ & $\begin{array}{l}-0.864 \\
-1.019 \\
-1.046 \\
-1.025\end{array}$ \\
\hline $\mathrm{H}_{2} \mathrm{O}$ & $\begin{array}{l}(D, T) \\
(T, Q) \\
(Q, 5) \\
(5,6)\end{array}$ & $\begin{array}{l}-299.4 \\
-299.4 \\
-298.8 \\
-298.2\end{array}$ & $\begin{array}{l}0.860 \\
0.860 \\
0.823 \\
0.740\end{array}$ & $\begin{array}{l}-292.5 \\
-298.9 \\
-298.7 \\
-298.1\end{array}$ & $\begin{array}{l}-0.864 \\
-0.987 \\
-0.980 \\
-0.934\end{array}$ \\
\hline $\mathrm{HF}$ & $\begin{array}{l}(D, T) \\
(T, Q) \\
(Q, 5) \\
(5,6)\end{array}$ & $\begin{array}{l}-314.9 \\
-314.9 \\
-315.1 \\
-314.2\end{array}$ & $\begin{array}{l}0.671 \\
0.671 \\
0.678 \\
0.608\end{array}$ & $\begin{array}{l}-303.8 \\
-314.1 \\
-314.9 \\
-314.1\end{array}$ & $\begin{array}{l}-0.864 \\
-1.018 \\
-1.036 \\
-0.996\end{array}$ \\
\hline $\mathrm{N}_{2}$ & $\begin{array}{l}(D, T) \\
(T, Q) \\
(Q, 5) \\
(5,6)\end{array}$ & $\begin{array}{l}-408.5 \\
-408.5 \\
-408.2 \\
-407.6\end{array}$ & $\begin{array}{l}0.843 \\
0.843 \\
0.826 \\
0.760\end{array}$ & $\begin{array}{l}-399.9 \\
-408.0 \\
-408.1 \\
-407.6\end{array}$ & $\begin{array}{l}-0.864 \\
-0.998 \\
-1.003 \\
-0.971\end{array}$ \\
\hline $\mathrm{Ne}$ & $\begin{array}{l}(D, T) \\
(T, Q) \\
(Q, 5) \\
(5,6)\end{array}$ & $\begin{array}{l}-315.6 \\
-315.6 \\
-316.4 \\
-315.7\end{array}$ & $\begin{array}{l}0.511 \\
0.511 \\
0.532 \\
0.497\end{array}$ & $\begin{array}{l}-300.8 \\
-314.6 \\
-316.2 \\
-315.6\end{array}$ & $\begin{array}{l}-0.864 \\
-1.035 \\
-1.065 \\
-1.044\end{array}$ \\
\hline
\end{tabular}

${ }^{a}\left(X_{1}, X_{2}\right)$ denotes the cardinal number pair used for extrapolation. ${ }^{b}$ Results obtained with the following constrained values of $\tau_{53}^{D T Q}$ : -0.932 for $\mathrm{CH}_{2} ;-1.288$ for $\mathrm{CO} ;-1.304$ for $\mathrm{F}_{2} ;-1.219$ for $\mathrm{H}_{2} \mathrm{O}$; -1.300 for $\mathrm{HF} ;-1.249$ for $\mathrm{N}_{2} ;-1.346$ for Ne. ${ }^{c}$ Ratios obtained when the following $\eta_{D D_{+} T}$ values are fixed (by fitting the $X=D, D_{+}, T$ energies): 0.813 for $\mathrm{CH}_{2} ; 1.979$ for $\mathrm{CO} ; 3.824$ for $\mathrm{F}_{2} ; 1.743$ for $\mathrm{H}_{2} \mathrm{O}$; 2.081 for $\mathrm{HF} ; 1.942$ for $\mathrm{N}_{2} ; 2.397$ for $\mathrm{Ne}$; see the text.

thermore, if these ratios are used for the extrapolations in $\mathrm{OH}$, one gets using the same units $-229.220 \pm 0.919(D, T)$, $-227.058 \pm 0.066(T, Q)$, and $-226.317 \pm 0.004(5,6)$, in slightly better agreement with the best estimate than USTE but slightly worse than GUSTE. Finally, one wonders about the error obtained if $\tau_{53}^{Q 56}(\mathrm{OH})$ were used for the extrapolations in $\mathrm{H}_{2} \mathrm{O}$. The results (in $\mathrm{mE}_{\mathrm{h}}$ ) are $-273.8(D, T),-296.4(T, Q)$, and $-298.3(Q, 5)$, in good agreement with the USTE values. Keeping in mind the slightly different level of correlation description used for $\mathrm{H}_{2} \mathrm{O}$ and $\mathrm{OH}$, two observations are in order from the above results. First, $\mathrm{H}_{2} \mathrm{O}$ is the only system where the GUSTE $(Q, 5)$ and USTE $(Q, 5)$ predictions differ by more than $0.1 \mathrm{mE}_{\mathrm{h}}$. This reinforces our belief that the $(Q, 5)$ extrapolated MRCI(Q) energy from eq 2 may be $1 \mathrm{mE}_{\mathrm{h}}$ or so in error due to a poor balanced level of description: a higher accuracy may require improvements on the basis or in the correlation description or both. Second, and perhaps most interesting, it suggests that in chemical reactions where the chemical bonds are broken (like $\mathrm{H}_{2} \mathrm{O}$ splitting into $\mathrm{OH}$ and $\mathrm{H}$ ), it is advantageous to choose $\tau_{53}$ somewhere in the middle between the reactant and the product values for the most balanced description of the PES using the GUSTE approach. Finding the optimal balance for the $A_{5} / A_{3}$ ratio in both systems (in this case, $\mathrm{H}_{2} \mathrm{O}$ and $\mathrm{OH}$ $+\mathrm{H})$ simultaneously may then be an important step for accurately describing a PES with GUSTE. 

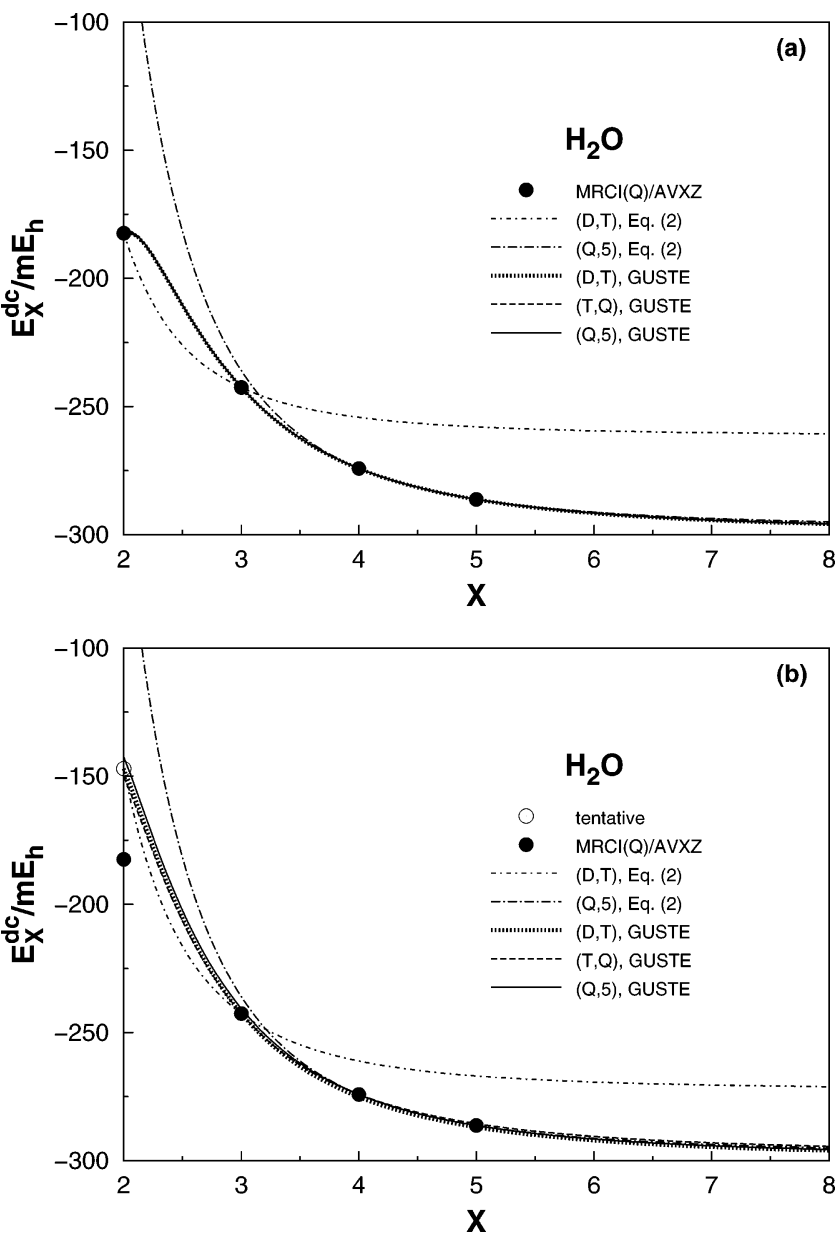

Figure 2. Extrapolations of the dynamical correlation energy for $\mathrm{H}_{2} \mathrm{O}$ : (a) using the calculated $\mathrm{MRCI}(\mathrm{Q}) / \mathrm{AVXZ}(X=D, T, Q, 5)$ energies; (b) as in (a) but using a tentative $\mathrm{MRCI}(\mathrm{Q}) / \mathrm{AVDZ}$ energy. For clarity, the curves referring to USTE will not be shown as they lie close to the GUSTE ones. See the text.

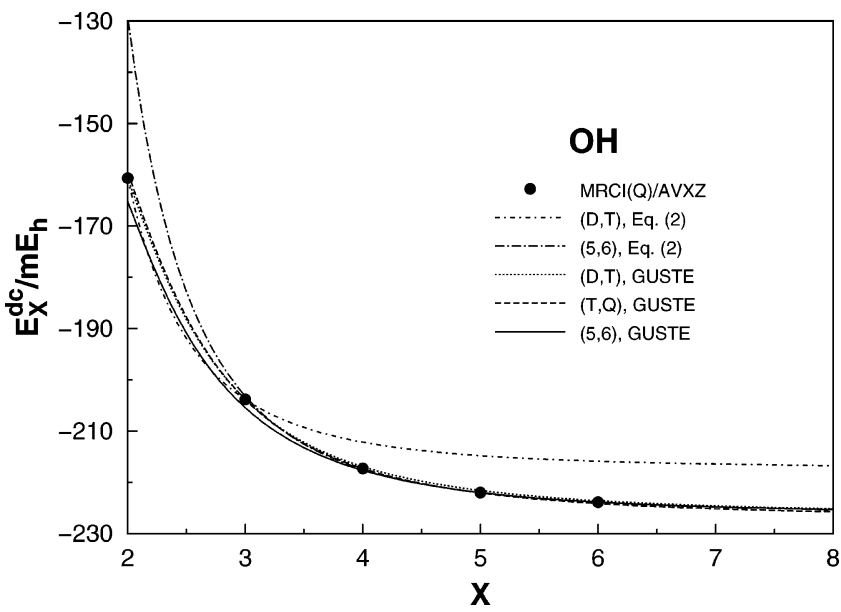

Figure 3. Extrapolations of the dynamical correlation energy for $\mathrm{OH}$ using the calculated MRCI $(\mathrm{Q}) / \mathrm{AVXZ}(X=D, T, Q, 5,6)$ energies. For clarity, the curves referring to USTE will not be shown as they lie close to the GUSTE ones. See the text.

A further remark is in order concerning GUSTE/DTQ. Although this requires only an extra calculation with $X=Q$, one wonders whether this third energy could be for the range $D-T$ or $2 \leq X \leq 3$. Since an $a b$ initio approach poses difficulties in specifying the basis set for a non-integer- $X$ calculation, the alternative is to predict the required energy from the results for $X=D$ and $T$ with an independent rule. For example, Truhlar ${ }^{17}$ has proposed the law $E_{X}=E_{\infty}+b / X^{2.4}$ optimized for $(D, T)$ extrapolations from CCSD and CCSD(T) energies. The extra point (denoted as $D_{+}$) may then be generated from such a rule, with $\tau_{53}^{D D_{+} T}$ and hence $\eta_{D D_{+} T}$ determined (jointly with $E_{\infty}$ and $A_{3}$ ) by fitting the $X=D, D_{+}, T$ energies to eqs 4 and 5. Two approaches may then be followed. The first consists of keeping fixed the scaling factor $\eta_{D D+T}$ for all extrapolations. Such an approach has been found ${ }^{55}$ to perform well for $\mathrm{He}_{2}$ using raw energies calculated with both the CC $[\mathrm{CCSD}$ and $\operatorname{CCSD}(\mathrm{T})]$ and $\mathrm{CI}(\mathrm{Q})$ methods. Indeed, it finds support in Figure 4, which shows that the values of $\eta_{D T Q}$ actually obtained by imposing $\tau_{53}^{D T Q}$ assume similar values for all extrapolations (note that the plotted scaling factors have been divided by their averaged value for all extrapolations in a given system). Except in one or two cases that justify the rule, the results obtained from the raw MP2, CCD, and CCSD energies deviate typically by 10 to $20 \%$, a fluctuation that may likely be absorbed by the $E_{\infty}$ and $A_{3}$ fitting parameters. Note that no extrapolation rule has been reported ${ }^{17}$ for $\operatorname{MRCI}(\mathrm{Q})$ energies. Given that a $X^{-2.4}$ dependence seems to yield acceptable results for $\mathrm{He}_{2}$ when using $\mathrm{CC}$ energies, this suggests that a similar power-law may, once the exponent is optimized, be also valid for $\mathrm{MRCI}(\mathrm{Q})$ energies. Indeed, if $\beta_{\mathrm{CI}}$ is defined from $X=D-$ 6 fits for the subset of systems studied ${ }^{27}$ by $\operatorname{MRCI}(\mathrm{Q})$ that overlaps with Klopper's ${ }^{22}$ one, namely $\mathrm{H}_{2} \mathrm{O}, \mathrm{HF}, \mathrm{N}_{2}, \mathrm{CO}$ and $\mathrm{F}_{2}$, the result will be $\beta_{\mathrm{CI}}=2.2$ (this value decreases to 1.9 if the fit covers only the $D-Q$ range of cardinal numbers).

To illustrate how the $\eta$-fixed method works, we have considered the seven systems studied in ref 22 with the CCSD/ VXZ method. However, rather than using the scaling values that mimic $\tau_{53}^{D T Q}$, we have utilized those that reproduce $\tau_{53}^{D D_{+} T}$, with the energy for $X=D_{+}$obtained by fitting Truhlar's ${ }^{17} X^{-2.4}$ law to Klopper's CCSD data for $X=D, T$. The results are collected in Table 9. For comparison, we give also in the third column of this table the extrapolated energies predicted from GUSTE/DTQ, where the ratio $A_{5} / A_{3}$ is constrained to be $\tau_{53}^{D T Q}$. Moreover, we list in column four the corresponding scaling factors $\eta_{D T Q}$, while column six reports the $\tau_{53}^{D D_{+} T}$ ratios predicted when $\eta_{D D_{+} T}$ is fixed at its optimum value in the fit to the $X=D, D_{+}, T$ energies. Although the extrapolated energies from GUSTE/ $D D_{+} T$ (in column five) show an improved accuracy when compared with those obtained from the traditional rule (2) (cf. Table 1), they are poorer than the GUSTE/ $D T Q$ or GUSTE/Q56 or GUSTE ones. In fact, the only exception is $\mathrm{CH}_{2}$, which is due to the fact that $\eta_{D D_{+} T} \sim \eta_{D T Q}$ for this system. Note that similar results are obtained if the value of $\tau_{53}^{D D_{+} T}$ associated with the $X=D, D_{+}, T$ energies $\left(\tau_{53}^{D D_{+} T}=\right.$ $-0.864)$ is imposed, rather than $\eta_{D D_{+} T}$. This is not surprising since $\eta_{D D_{+} T}$ and $\tau_{53}^{D D_{+} T}=-0.864$ are equivalent attributes of Truhlar's ${ }^{17}$ law. Despite the somewhat modest results, it is interesting to note that the GUSTE/ $D D_{+} T$ rule employs raw energies only up to $X=T$ but shows a performance better than the traditional law (2) when using the same cardinal numbers for the extrapolation.

\section{Concluding Remarks}

We summarize a few properties that the GUSTE rule here developed satisfies:

1. It extrapolates reliably the (full or dynamical) correlation energy to the CBS limit, irrespectively of the cardinal numbers used for the extrapolation.

2. Once calibrated the scheme is dual-level as it requires only two ab initio calculations with distinct basis sets for extrapolating the correlation energy. 

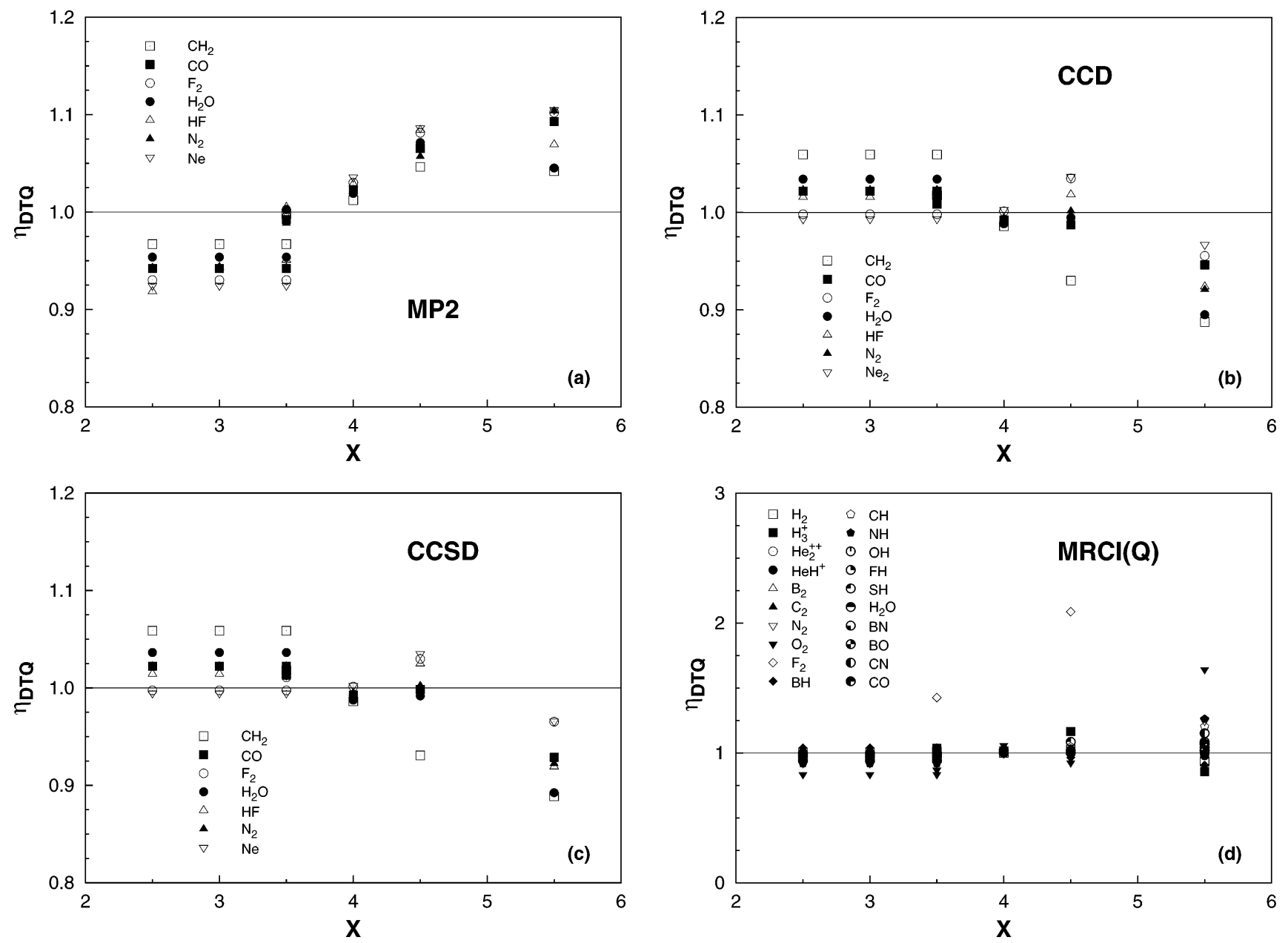

Figure 4. Values of $\eta_{D T Q}$ obtained by diving the actually calculated results by the average value obtained in the $(D, T),(D-Q),(T, Q),(D-5)$, $(Q, 5),(D-6)$, and $(5,6)$ extrapolations: (a) MP2; (b) CCD; (c) CCSD; (d) MRCI(Q).

3. The calibration requires the least possible amount of calculations with high cardinal numbers at a single geometry (without exceeding $X=Q$ in GUSTE/DTQ), although the accuracy of the method is enhanced when such data is available.

4. The calibration does not utilize data alien to the raw $a b$ initio energies (information from other laws may, however, be utilized as discussed in section 3).

5. The method reduces to the original USTE scheme when $\eta$ $=1$ in eq 8 .

In summary, we have analyzed generalizations of the USTE rule that can improve even further the accuracy of the extrapolated energies, especially when using $X=D$ and $T$ basis sets. Such generalizations are based on the premise that the ratio of the triplet-pair (see section 2) to singlet-pair contributions does not vary significantly over the configuration space of the molecule. Work to test this assertion would be valuable though conceptually difficult at regions where more than one electronic configuration plays a role. Finally, the new scheme affords enough flexibility to treat different singlet/triplet-pair situations, and hence is expected to be valid for both ground and excited electronic states. This may help to enhance the development of methods ${ }^{50-52}$ that allow the calculation of accurate potential energy surfaces at an unprecedented low computational cost. Parallel to this, it may open the way for calculating molecular properties that may be sensitive to small energy differences and therefore to quality of the treatment of electron correlation. In particular, its use for studying static electric response properties of molecules or groups or molecules, which are formally developed via energy derivatives and hence are intrinsic to features of electronic structure, ${ }^{58}$ may be worth pursuing in future work.

Acknowledgment. This work is supported by Fundação para a Ciência e a Tecnologia, Portugal (Contracts POCI/QUI/60501/ 2004, POCI/AMB/60261/2004, and REEQ/128/QUI/2005), under the auspices of POCI 2010 of Quadro Comunitário de Apoio III co-financed by FEDER.

\section{References and Notes}

(1) Kato, T. Commun. Pure Appl. Math. 1957, 10, 151.

(2) Pack, R. T.; Brown, W. B. J. Chem. Phys. 1966, 45, 556.

(3) Anderson, J. B.; Traynor, C. A.; Boghosian, B. M. J. Chem. Phys. 1993, 99, 345.

(4) Korona, T.; Williams, H.; Bukowski, R.; Jeziorski, B.; Szalewicz, K. J. Chem. Phys. 1997, 106, 5109.

(5) Komasa, J. J. Chem. Phys. 1999, 110, 7909.

(6) Klopper, W.; Manby, F. R.; Ten-No, S.; Valeev, E. F. Int. Rev. Phys. Chem. 2006, 3, 427.

(7) Tew, D. P.; Klopper, W.; Neiss, C.; Hättig, C. Phys. Chem. Chem. Phys. 2007, 9, 1921.

(8) Helgaker, T.; Jørgensen, P.; Olsen, J. Molecular ElectronicStructure Theory; Wiley: Chichester, 2000.

(9) Piecuch, P.; Włoch, M.; Varandas, A. J. C. Theor. Chem. Acc. (in press).

(10) Cardonen, W.; Gdanitz, R. J. J. Chem. Phys. 2005, 123, 024304

(11) Dunning, T. H., Jr. J. Chem. Phys. 1989, 90, 1007.

(12) Kendall, R. A.; Dunning, T. H., Jr.; Harrison, R. J. J. Chem. Phys. 1992, 96, 6769.

(13) Woon, D. E.; Dunning, T. H., Jr. J. Chem. Phys. 1995, 103, 4572. 
(14) Dunning, T. H., Jr.; Peterson, K. A.; Woon, D. E. Encyclopedia of Computational Chemistry; v. R.; Schleyer, P.; Allinger, N. L.; Clark, T.; Gesteiger, J.; Kolman, P. A.; Schaefer, H. F., III, Eds. Wiley: Chichester 1998, p 88.

(15) Helgaker, T.; Klopper, W.; Koch, H.; Noga, J. J. Chem. Phys. 1997, $106,9639$.

(16) Halkier, A.; Helgaker, T.; Jørgensen, P.; Klopper, W.; Koch, H.; Olsen, J.; Wilson, A. K. Chem. Phys. Lett. 1998, 286, 243.

(17) Truhlar, D. G. Chem. Phys. Lett. 1998, 294, 45.

(18) Fast, P. L.; Sánchez, M. L.; Truhlar, D. G. J. Chem. Phys. 1999, 111,2921

(19) Halkier, A.; Helgaker, T.; Jørgensen, P.; Klopper, W.; Olsen, J. Chem. Phys. Lett. 1999, 302, 437.

(20) Varandas, A. J. C. J. Chem. Phys. 2000, 113, 8880.

(21) Gdanitz, R. J. J. Chem. Phys. 2000, 113, 5145.

(22) Klopper, W. Mol. Phys. 2001, 6, 481.

(23) Klopper, W. J. Chem. Phys. 2001, 115, 761.

(24) Valeev, E. F.; Allen, W. D.; Hernandez, R.; Sherrill, C. D.; Schaefer,

H. F., III. J. Chem. Phys. 2003, 118, 8594.

(25) Huh, S. B.; Lee, J. S. J. Chem. Phys. 2003, $118,3035$.

(26) Schwenke, D. W. J. Chem. Phys. 2005, 122, 014107.

(27) Varandas, A. J. C. J. Chem. Phys. 2007, 126, 244105.

(28) Bakowies, D. J. Chem. Phys. 2007, 127, 084105.

(29) Schwartz, C. Phys. Rev. 1962, 126, 1015.

(30) Schwartz, C. Methods Comput. Phys. 1963, 2, 241

(31) Byron, F. W.; Joachain, C. J. Phys. Rev. 1967, 157, 1.

(32) Bunge, C. F. Theor. Chim. Acta 1970, 16, 126.

(33) Carroll, D. P.; Silverstone, H. J.; Metzger, R. M. J. Chem. Phys. 1979, 71, 4142 .

(34) Morrell, G. O.; Parr, R. G. J. Chem. Phys. 1979, 71, 4139

(35) Nyden, M. R.; Petersson, G. A. J. Chem. Phys. 1981, 75, 1843.
(36) Schmidt, H. M.; von Hirschhausen, H. Phys. Rev. A 1983, 28, 3179.

(37) Hill, R. N. J. Chem. Phys. 1985, 83, 1173.

(38) Salomonson, S.; Öster, P. Phys. Rev. A 1989, 40, 5559.

(39) Kutzelnigg, W.; Morgan, J. D., III. J. Chem. Phys. 1992, 96, 4484

(40) Schmidt, H. M.; Linderberg, J. Phys. Rev. A 1994, 49, 4404.

(41) Flores, J. R.; Slupski, R.; Jankowski, K. J. Chem. Phys. 2006, 124, 104107

(42) Gordon, M. S.; Nguyen, K. A.; Truhlar, D. G. J. Phys. Chem. 1989, 93, 7356 .

(43) Peterson, K. A.; Dunning, T. H., Jr. J. Chem. Phys. 1997, 106, 4119.

(44) Peterson, K. A.; Xantheas, S. S.; Dixon, D. A.; Dunning, T. H., Jr. J. Phys. Chem. A 1998, 102, 2449.

(45) Feller, D.; Peterson, K. A.; Crawford, T. D. J. Chem. Phys. 2006 124, 054107.

(46) Feller, D.; Sordo, J. A. J. Chem. Phys. 2000, 113, 485.

(47) Klopper, W.; Bak, K. L.; Jørgensen, P.; Olsen, J.; Helgaker, T. J. Phys. B: At. Mol. Opt. Phys. 1999, 32, R103.

(48) Karton, A.; Martin, J. M. L. Theor. Chem. Acc. 2006, 115, 330.

(49) Varandas, A. J. C. Phys. Scr. (Comm. At. Opt. Mol. Phys.) 2007, $76, \mathrm{C} 28$

(50) Varandas, A. J. C.; Piecuch, P. Chem. Phys. Lett. 2006, 430, 448.

(51) Varandas, A. J. C. Chem. Phys. Lett. 2007, 443, 398.

(52) Varandas, A. J. C. J. Chem. Phys. 2007, 127, 114316.

(53) Klopper, W.; Samson, C. C. M. J. Chem. Phys. 2002, 116, 6397.

(54) Bytautas, L.; Ruedenberg, K. J. Chem. Phys. 2005, 122, 154110.

(55) Varandas, A. J. C. Acc. Theor. Chem. (submitted for publication).

(56) Wolniewicz, L. J. Phys. B: At. Mol. Opt. Phys. 1999, 32, 2257.

(57) Polyansky, O. L.; Császar, A. G.; Shirin, S. V.; Nikolai, F.; Barletta,

P.; Tennyson, J.; Schwenke, D. W.; Knowles, P. J. Science 2003, 299, 539.

(58) Dykstra, C. E. J. Mol. Struct. Theochem 2001, 573, 63. 